\title{
INDEX TO THE BIBLIOGRAPHY, SUBJECTS AND COUNTRIES
}

GENERAL ISSUES

Religions and Philosophy, 277 446

Socia Science, 128ff., 277ff., 446ff. History, 132ff., 282ff., $450 \mathrm{ff}$.

Contemporary Issues, 136, 286f., $455 f$.

CONTINENTS AND COUNTRIES

Africa, 136f., 288

Algeria, 137

Ethiopia, 456

Nigeria, 288

South Africa, 137

Uganda, 289
America, 137, 456

Argentina, 289

Brazil, 289f., 457

Canada, 290, 457f.

Chile, 290

Trinidad and Tobago, 138

United States of America, 138f., 290ff., 458f.

Asia, 459

Ceylon, 139

China, 140, 295f., $459 \mathrm{ff}$.

India, 140, 296, 461

Indonesia, 140f., $296 f$., $461 f$.

Iran, 141

Japan, 141, 297

Laos, 462

Turkey, 297

Europe, $462 \mathrm{f}$.

Austria, 141f., 298, $463 f$.
Belgium, 142, 465

Czechoslovakia, 142

Eire - Ireland, 142f., 298

France, 143ff., 298ff., 465ff.

Germany, 145ff., 301ff., 468ff.

Great Britain, 151ff., 310ff., $472 \mathrm{ff}$.

Greece, 156, $319 f$.

Hungary, 156

Italy, 156f., 320f., 477ff.

The Netherlands, $157,321,480$

Norway, $480 f$.

Portugal, 157f.

Spain, 158, 322f., 481f.

Sweden, 158

Switzerland, 158

Union of Socialist Soviet Republics - Russia, 159ff., 323f., 482ff.

Yugoslavia, 162 